\title{
NPR1 is Instrumental in Priming for the Enhanced flg22-induced MPK3 and MPK6 Activation
}

\author{
So Young Yi*, Sung Ran Min and Suk-Yoon Kwon* \\ Plant Systems Engineering Research Center, KRIBB, Daejeon 305-806, Korea \\ (Received on October 26, 2014; Revised on March 5, 2015; Accepted on March 15, 2015)
}

Pathogen-associated molecular patterns (PAMPs) activate mitogen-activated protein kinases (MAPKs), essential components of plant defense signaling. Salicylic acid (SA) is also central to plant resistance responses, but its specific role in regulation of MAPK activation is not completely defined. We have investigated the role of SA in PAMP-triggered MAPKs pathways in Arabidopsis SA-related mutants, specifically in the fig22-triggered activation of MPK3 and MPK6. cim6, sid2, and npr1 mutants exhibited wild-type-like flg22-triggered MAPKs activation, suggesting that impairment of SA signaling has no effect on the flg22-triggered MAPKs activation. Pretreatment with low concentrations of SA enhanced flg22-induced MPK3 and MPK6 activation in all seedlings except $n p r 1$, indicating that NPR1 is involved in SA-mediated priming that enhanced flg22induced MAPKs activation.

Keywords : CIM6, flg22 response, mitogen-activated protein kinases (MAPKs), MPK3, MPK6, NPR1, priming, salicylic acid signaling, SID2

In Arabidopsis, perception of the bacterial protein, flagellin, occurs at the plasma membrane through the FLAGELLINSENSITIVE 2 (FLS2) and BRI1-ASSOCIATED KINASE 1 (BAK1) complex (Gomez-Gomez and Boller, 2000; Chinchilla et al., 2007). FLS2 binds to flg22, a 22-amino acid flagellin-derived peptide that is a pathogen associated molecular pattern (PAMP). Once bound to flg22, the complex initiates several defense responses, including calcium flux, activation of several MAPKs, an oxidative burst, and

*Co-corresponding authors.

So Young Yi

Phone) +82-42-860-4433, FAX) +82-42-860-4608

E-mail) yisy@dgist.ac.kr

Suk-Yoon Kwon

Phone) +82-42-860-4340, FAX) +82-42-860-4349

E-mail)sykwon@kribb.re.kr induction of ethylene and SA biosynthesis. These responses are involved in triggering the intermediate and late defense responses, including activation of a diverse array of defense genes, callose deposition, and inhibition of seedling growth (Boller and Felix, 2009; Tsuda et al., 2008).

MAPK cascades are signaling pathways downstream of sensors or receptors that transduce extracellular stimuli into intracellular responses in eukaryotes. The Arabidopsis MAPKs, MPK3, MPK4 and MPK6, are activated by flg22 treatment within five minutes (Asai et al., 2002; Droillard et al., 2004). Activation of the MAPK cascade positively regulates the expression of several defense-related genes and confers resistance to plant pathogens (Asai et al., 2002). MPK3 and MPK6 are pivotal in systemic acquired resistance (SAR) and SA-mediated priming of plants for disease resistance (Beckers et al., 2009).

The flg22-triggered oxidative burst and induction of transcription of the gene for WRKY29 and the gene for the flg22-induced receptor-like protein FRK1, which are early responses, have been shown to depend on SA signaling (Yi et al., 2014). We wanted to know if SA signaling also has a role in a very early flg22 response, MAPK activation. We treated wild-type (WT) Arabidopsis seedlings and several SA-related mutants with SA or flg22 and assessed dual phosphorylation of the TEY motif of MAPK. The plants used were WT and the mutants, sid2, which has low SA content (Nawrath and Metraux, 1999); cim6, which has high SA content (Maleck et al., 2002); and npr1, which has a block in SA signaling (Cao et al., 1994). As is shown in Figure 1, when plants were treated with SA, no TEY phosphorylation of MPK3 and MPK6 was detected in any of the seedlings, whereas it was observed after flg22 treatment. Recent report showed that during development of benzothiadiazole (BTH; chemical analogue of SA) induced resistance in Arabidopsis and the priming is associated with accumulation of mRNA and inactive proteins of MPK3 and MPK6 (Beckers et al., 2009). In other words, SA or BTH involved in the accumulation of unphosphorylated MPK3/6 but not in activation of MPK3/6. 
It has been suggested that these MAPKs function downstream of reactive oxygen species (ROS) in plant immunity, because they are activated by exogenous $\mathrm{H}_{2} \mathrm{O}_{2}$ (Colcombet and Hirt, 2008). Because cim6 and sid2 mutant seedlings are reported to have altered flg22-triggered ROS production (Yi et al., 2014), we expected altered flg22-induced MAPK activation in these mutants. However, all of the mutants exhibited WT-like flg22-induced activation of MAPKs (Fig. 1). Mersmann and colleagues also observed an impaired oxidative burst accompanied by WT-like activation of MPK3 and MPK6 in flg22-stimulated ethyleneinsensitive mutants (Mersmann et al., 2010). Based on our results, we suggest that the flg22-triggered oxidative burst and activation of MPK3/MPK6 are independent early signaling events in plant immunity, acting downstream of FLS2.

Flg22 perception by FLS2 leads to the induction of transcription of WRKY29 and FRK1. This induction occurs through the activation of a MAPK signaling cascade consisting of MEKK1, MKK4/MKK5 and MPK3/MPK6 (Asai et al., 2002). Transcript levels of WRKY29 and FRK1 were reduced by approximately $50 \%$ in sid 2 compared with WT, one hour after flg22 treatment (Yi et al., 2014). However, sid2 showed WT-like flg22-triggered MPK3 and

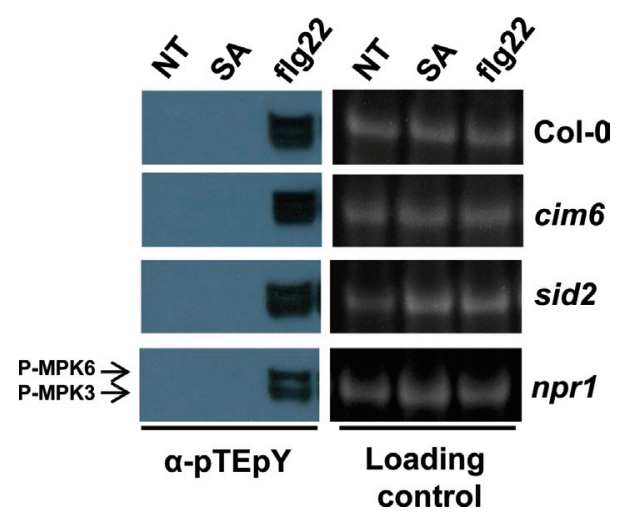

Fig. 1. SA-related mutants exhibit WT-like flg22-induced phosphorylation of MPK6 and MPK3. WT plant and SA-related mutants (cim6, nprl and sid2) were treated with SA $(100 \mu \mathrm{M})$ for $24 \mathrm{~h}$ or flg2 $2(1 \mu \mathrm{M})$ for $10 \mathrm{~min}$. Seedlings were then assayed for dual phosphorylation of the TEY motif in MPK3 and MPK6. Phosphorylated MAPKs corresponding to MPK3 and MPK6 is indicated. NT is representing control $\left(\mathrm{H}_{2} \mathrm{O}\right)$ treated seedling. Activated MAPKs were detected by immunoblot using the antibody against Phospho-p44/42 MAPK (Erk1/2) (Cell Signaling Technology). Signal detection was performed using the enhanced chemiluminescence (ECL) western blotting detection kit (Bio-Rad). The experiment was performed three times with similar results. Prior to transfer to PVDF membrane, protein equal loading was checked by fluorescence image of Rubisco in the stain-free gels.
MPK6 activation (Fig. 1). These studies suggest that flg22induced WRKY29 and FRK1 transcription are regulated by SA signaling as well as activated MPK3 and MPK6 signaling, but two signaling act independently.

Priming with low concentrations of SA or BTH has been reported to increase resistance to subsequent pathogen infection, as evidenced by faster and greater expression of defense genes compared to non-primed plants (Prime et al., 2006). It has also been shown that BTH pre-treatment induced the accumulation of unphosphorylated, inactive forms of MPK3 and MPK6 in Arabidopsis, but when these primed plants were stressed, they accumulated more phosphorylated, active MPK3 and MPK6 than unstressed plants. This enhanced accumulation correlated with greater expression of $P A L$, which codes for phenylalanine ammonia lyase, and of $P R-1$, which codes for the pathogenesisrelated protein 1 (Beckers et al., 2009). The potentiated responses to pathogen infection, wounding, or infiltration of water into leaves that result from priming with SA or BTH require expression of the NPR1/NIM1 gene in Arabidopsis (Kohler et al., 2002). NPR1 is also involved in SA-induced priming for enhancing flg22 responses, the oxidative burst, the induction of WRKY29 and FRK1 transcription, and callose deposition (Yi et al., 2014). To determine whether NPR1 is also important in priming for enhanced flg22triggered MAPKs activation, we analyzed the dual TEY phosphorylation in WT and SA-related mutants (Fig. 2).

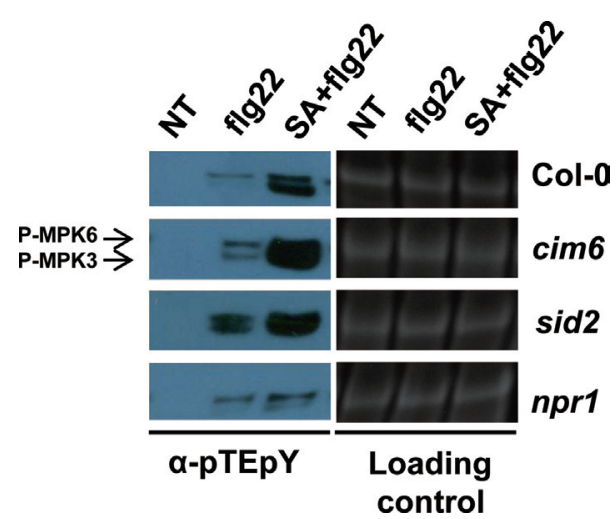

Fig. 2. The phosphorylation of MPK3 and MPK6 is enhanced in SA pretreated seedlings but $n p r l$ showed reduction of potentiated dual phosphorylation. Arabidopsis seedlings were pre-incubated with SA $(100 \mu \mathrm{M})$ or $\mathrm{H}_{2} \mathrm{O}$ for $24 \mathrm{~h}$ before the treatment of flg22 $(1 \mu \mathrm{M})$. Seedlings were then assayed for dual phosphorylation of the TEY motif in MPK3 and MPK6 10 minute after treatment with flg22. Activated MAPKs were detected by immunoblot blot using the antibody against Phospho-p44/42 MAPK (Erk1/2). The experiment was performed three times with similar results. Prior to transfer to PVDF membrane, protein equal loading was checked by fluorescence image of Rubisco in the stain-free gels. 
The results show that pretreatment with SA served as an flg22-signaling enhancer in WT, cim6, and sid2 seedlings. The nprl seedlings, however, did not show SA-dependent enhancement of the flg22-triggered MAPK activation, indicating that NPR1 is instrumental in priming for stronger activation of MPK3 and MPK6. Interestingly, MPK3 activation is stronger than MPK6 in Arabidopsis seedlings treated with SA+flg22. However, both MPK3 and MPK6 seem to be important for full priming in Arabidopsis. This may explain why knockout of any one of the two genes encoding MPK3 or MPK6 doesn't always attenuate induced resistance (Beckers et al., 2009).

Our results from a previous study (Yi et al., 2014) suggest that SA acts at an early stage of the flg22 response, on the oxidative burst that may be involved in callose deposition, a late flg22 response. Our current results suggest that impaired SA signaling has no effect on the flg22-triggered MAPK activation. It may be that the flg22-triggered oxidative burst and MPK3 and MPK6 activation are two independent, early signaling events in plant immunity, acting downstream of FLS2. Moreover, we provide evidence that NPR1 is involved in SA-dependent priming for enhancing the flg22-induced MPK3/MPK6. Based on previous reports (Beckers et al., 2009; Conrath et al., 2002; Kohler et al., 2002; Yi et al., 2014) as well as on these findings, we propose that NPR1-mediated priming of defense responses is also important in flg22 responses.

\section{Acknowledgments}

This work was supported by the Technology Development Program for Agriculture and Forestry (610008-05-5-SB210 and 112127-05-3-HD030 to SYK), the KRIBB Research Initiative Program and Golden Seed Project (2013003-041-WTD21 to SRM) funded by the Ministry of Agriculture, Food and Rural Affairs, Republic of Korea.

\section{References}

Asai, T., Tena, G., Plotnikova, J., Willmann, M. R., Chiu, W. L., Gomez-Gomez, L., Boller, T., Ausubel, F. M. and Sheen, J. 2002. Map kinase signalling cascade in Arabidopsis innate immunity. Nature 415:977-983.

Beckers, G. J., Jaskiewicz, M., Liu, Y., Underwood, W. R., He, S. Y., Zhang, S. and Conrath, U. 2009. Mitogen-activated protein kinases 3 and 6 are required for full priming of stress responses in Arabidopsis thaliana. Plant Cell 21:944-953.

Boller, T. and Felix, G. 2009. A renaissance of elicitors: Perception of microbe-associated molecular patterns and danger sig- nals by pattern-recognition receptors. Annu. Rev. Plant Biol. 60:379-406.

Cao, H., Bowling, S. A., Gordon, A. S. and Dong, X. 1994. Characterization of an Arabidopsis mutant that is nonresponsive to inducers of systemic acquired resistance. Plant Cell 6:15831592.

Chinchilla, D., Zipfel, C., Robatzek, S., Kemmerling, B., Nurnberger, T., Jones, J. D., Felix, G. and Boller, T. 2007. A flagellin-induced complex of the receptor FLS2 and BAK1 initiates plant defence. Nature 448:497-500.

Colcombet, J. and Hirt, H. 2008. Arabidopsis MAPKs: A complex signalling network involved in multiple biological processes. Biochem. J. 413:217-226.

Conrath, U., Pieterse, C. M. and Mauch-Mani, B. 2002. Priming in plant-pathogen interactions. Trends Plant Sci. 7:210-216.

Droillard, M. J., Boudsocq, M., Barbier-Brygoo, H. and Lauriere, C. 2004. Involvement of MPK4 in osmotic stress response pathways in cell suspensions and plantlets of arabidopsis thaliana: Activation by hypoosmolarity and negative role in hyperosmolarity tolerance. FEBS Lett. 574:42-48.

Gomez-Gomez, L. and Boller, T. 2000. Fls2: An lrr receptor-like kinase involved in the perception of the bacterial elicitor flagellin in Arabidopsis. Mol. Cell 5:1003-1011.

Kohler, A., Schwindling, S. and Conrath, U. 2002. Benzothiadiazole-induced priming for potentiated responses to pathogen infection, wounding, and infiltration of water into leaves requires the npr1/nim1 gene in Arabidopsis. Plant Physiol. 128:1046-1056.

Maleck, K., Neuenschwander, U., Cade, R. M., Dietrich, R. A., Dangl, J. L. and Ryals, J. A. 2002. Isolation and characterization of broad-spectrum disease-resistant Arabidopsis mutants. Genetics 160:1661-1671.

Mersmann, S., Bourdais, G., Rietz, S. and Robatzek, S. 2010. Ethylene signaling regulates accumulation of the FLS2 receptor and is required for the oxidative burst contributing to plant immunity. Plant Physiol. 154:391-400.

Nawrath, C. and Metraux, J. P. 1999. Salicylic acid inductiondeficient mutants of Arabidopsis express PR-2 and PR-5 and accumulate high levels of camalexin after pathogen inoculation. Plant Cell 11:1393-1404.

Prime, A. P. G., Conrath, U., Beckers, G. J., Flors, V., GarciaAgustin, P., Jakab, G., Mauch, F., Newman, M. A., Pieterse, C. M., Poinssot, B., Pozo, M. J., Pugin, A., Schaffrath, U., Ton, J., Wendehenne, D., Zimmerli, L. and Mauch-Mani, B. 2006. Priming: Getting ready for battle. Mol. Plant-Microbe Interact. 19:1062-1071.

Tsuda, K., Glazebrook, J. and Katagiri, F. 2008. The interplay between mamp and sa signaling. Plant Signal Behav. 3:359-361.

Yi, S. Y., Shirasu, K., Moon, J. S., Lee, S. G. and Kwon, S. Y. 2014. The activated sa and ja signaling pathways have an influence on flg22-triggered oxidative burst and callose deposition. PLoS One 9:e88951. 